\title{
Uso do aparelho intraoral no controle da apneia obstrutiva do sono: relato de caso
}

\author{
Use of the intraoral device in the control of the obstructive sleep apnea: case report
}

Uso del aparato intraoral en el control de la apnea obstructiva del sueño: reporte de caso

Jeferson Batista Santiago ${ }^{1 *}$, Olívia Augusta Araújo Dias ${ }^{1}$, Joana de Ângelis Alves Silva ${ }^{1}$, Mayara Monique Silva de Oliveira ${ }^{1}$, Josué Alves ${ }^{1}$, Irany Porto Gurgel do Amaral ${ }^{1}$, Maria Flávia de Paiva Siqueira ${ }^{1}$, Paulo Correia Barbosa Filho ${ }^{2}$, Thayane Celina Silva Lessa ${ }^{1}$, Thaís Emmanuelle Melo da Silva ${ }^{1}$.

\section{RESUMO}

Objetivo: Relatar o uso de aparelho intraoral (AIO) no controle da apneia obstrutiva do sono (AOS). Detalhamento do caso: Paciente de 55 anos, gênero masculino, compareceu ao consultório odontológico por encaminhamento de um médico otorrinolaringologista, relatando ter a AOS e não conseguir aderir a terapia com o Continuous Positive Airway Pressure (CPAP) por achá-lo incômodo de usar. Durante o período de adaptação do AIO não houve queixas, e o paciente relatou melhoras dos sinais e sintomas que possuía, tais como: ronco, sonolência diurna e cefaléia. Após um ano e três meses de tratamento, de acordo com os resultados das polissonografias e o registro do questionário, o paciente havia normalizado os parâmetros polissonográficos analisados no estudo, concretizando o controle da AOS pelo uso do AIO. Considerações finais: Alguns tratamentos podem alcançar o controle da AOS, devolvendo ao indivíduo uma boa qualidade de vida. Para os pacientes com indicação de uso (apneia leve a moderada) e que conseguem aderir ao tratamento com o AIO, este é considerado um método eficaz no controle da síndrome, proporcionando mais conforto durante o sono, dessa forma consolidando o sucesso da terapia.

Palavras-chave: Síndromes da apneia do sono, Transtornos do sono-vigília, Sono.

\section{ABSTRACT}

Objective: To report the use of an intraoral device (IOD) to control obstructive sleep apnea (OSA). Case details: 55-year-old male patient came to the dental office by referral from an otolaryngologist, reporting having OSA and not being able to adhere to the Continuous Positive Airway Pressure (CPAP) therapy because he found it uncomfortable to use. During the IOD adaptation period, there were no complaints, and the patient reported improvements in the signs and symptoms he had, such as: snoring, daytime sleepiness and headache. After one year and three months of treatment, according to the results of the polysomnography and the registration of the questionnaire, the patient had normalized the polysomnographic parameters analyzed in the study, realizing the control of OSA by the use of the IOD. Final considerations: Some treatments can achieve OSA control, giving the individual a good quality of life. For patients with indication for use (mild to moderate apnea) and who are able to adhere to the treatment with IOD, this is considered an effective method in controlling the syndrome, providing more comfort during sleep, thus consolidating the success of the therapy.

Keywords: Sleep apnea syndromes, Sleep wake disorder, Sleep.

\section{RESUMEN}

Objetivo: Informar el uso de un aparato intraoral (AO) para controlar la apnea obstructiva del sueño (AOS). Detalles del caso: Paciente masculino de 55 años asistió al consultorio dental por recomendación de un otorrinolaringólogo, informando que tenía AOS y que no pudo adherirse a la terapia de presión positiva continua en las vías respiratorias (CPAP) porque le resultaba incómodo de usar. Durante el período de adaptación con el $\mathrm{AO}$, no hubo quejas, y el paciente informó mejoras en los signos y síntomas que tenía, como: ronquidos, somnolencia diurna y dolor de cabeza. Después de un año y tres meses de tratamiento, de

${ }^{1}$ Universidade de Pernambuco (UPE), Camaragibe - PE. *E-mail: jefersonbatistas@hotmail.com

2 Universidade de Pernambuco (UPE), Recife - PE. 
acuerdo con los resultados de la polisomnografía y el registro del cuestionario, el paciente había normalizado los parámetros polisomnográficos analizados en el estudio, realizando el control de AOS mediante el uso del AO. Consideraciones finales: Algunos tratamientos pueden lograr el control de AOS, dando al individuo una buena calidad de vida. Para los pacientes con indicación de uso (apnea leve a moderada) y que pueden adherirse al tratamiento con $\mathrm{AO}$, este se considera un método eficaz para controlar el síndrome, brindando más comodidad durante el sueño, consolidando así el éxito de la terapia.

Palabras clave: Síndromes de la Apnea del Sueño, Trastornos del Sueño-Vigilia, Sueño.

\section{INTRODUÇÃO}

O sono pode ser definido como sendo um conjunto de alterações comportamentais e fisiológicas que acontecem em conjunto e se associam a atividades elétricas cerebrais características. Neste estado existe uma postura relaxada, um elevado limiar para resposta a estímulos externos e atividade motora reduzida ou ausente (NEVES GSML, et al., 2013).

Geralmente durante o sono microdespertares são ocasionados por alguns estímulos que não causam desconforto suficiente para nos manterem acordados, os quais levam à uma má qualidade do sono. Um dos distúrbios mais frequentes do sono é a Apneia obstrutiva do sono (AOS) (PACHECO FYR, et al., 2016).

A AOS é um distúrbio comum do sono, e tem como características o colapso recorrente das vias aéreas superiores durante o mesmo, ocasionando a fragmentação do sono e a dessaturação do oxigênio (SUTHERLAND K, et al., 2014). Classifica-se a AOS segundo o índice de apneia e hipopneia (IAH), o qual toma-se como referência a frequência de eventos respiratórios durante o sono, sendo leve (IAH entre 5 e 15 eventos/hora), moderada (IAH entre 15 e 30 eventos/hora) ou grave (IAH > 30 eventos/hora), proporcionando uma melhor previsibilidade acerca das complicações que podem ocorrer a longo prazo, permitindo que haja um melhor direcionamento para o tratamento (CAMPOSTRINI DDA, et al., 2014).

O exame padrão ouro para o diagnóstico de apneia obstrutiva do sono é a polissonografia (PSG). A PSG é um exame noturno onde são registrados diferentes sinais fisiológicos, tais como o eletroencefalograma (EEG), eletrooculograma (EOG), eletromiograma (EMG), eletrocardiograma (ECG), fluxo de ar, saturação de oxigênio no sangue arterial, esforços respiratórios, roncos e posição do corpo, entre outros (DEY D, et al., 2018). Alguns dos parâmetros a serem observados na PSG são: o grau de severidade da apneia pelo IAH (Índice de apneia e hipopneia) por hora de sono e a saturação de oxihemoglobina que deve estar acima de 90\% (ALENCAR HBB, et al., 2016; GODOLFIM LR, 2010).

Se o paciente for diagnosticado com o transtorno, pode-se intervir no controle do mesmo com o objetivo de devolver o bem-estar e a qualidade de vida ao paciente. Entre as terapias disponíveis, existem duas que possuem um alto grau de sucesso no controle da AOS, a utilização da máscara nasal - CPAP (Continuous Positive Airway Pressure) e a utilização de aparelhos intra orais (AIOs) (ITO FA, et al., 2005).

A pressão positiva contínua nas vias aéreas (CPAP em inglês, continuous positive airway pressure) é considerado o tratamento padrão ouro para a AOS e este melhora os sintomas e as alterações cardiometabólicas do paciente (GUIMARÃES MLR, et al., 2018). O CPAP atua, basicamente, aspirando ar do meio ambiente, filtrando e enviando-o ao paciente através de um tubo flexível. $O$ colapso das paredes musculares faringeanas é evitado por conta do ar sob pressão que penetra nas vias aéreas, prevenindo a ocorrência das apneias e hipopnéias (SILVA GA e PACHITO DV, 2006).

O aparelho de avanço mandibular (AAM) é o tipo de AIO mais utilizado no tratamento da AOS, age protruindo a mandíbula e aumentando o tamanho das vias aéreas superiores(SAGLAM-AYDINATAY B e TANER T, 2018). O colapso entre os tecidos da orofaringe e da base da língua é prevenido por conta dessa distensão, evitando desta forma o fechamento da via aérea superior, isto pode ser observado por meio de imagens e por videoendoscopia (BITTENCOURT LRA, et al., 2009).

Esse estudo tem como obejtivo verificar, a partir de um relato de caso, a eficácia do AIO no controle da AOS em um paciente que não conseguiu se adaptar a terapia com o CPAP. Foram realizados exames polissonográficos para monitorar as mudanças fisiológicas durante o sono e um questionário para avaliar a opinião do paciente quanto ao uso do aparelho intra oral em comparação com o CPAP. 


\section{RELATO DE CASO}

Seguindo os preceitos estabelecidos pela resolução 466/2012 do conselho Nacional de Saúde (CNS), o presente estudo foi aprovado pelo comitê de ética em Pesquisa da Universidade de Pernambuco (UPE) CAAE: 98303718.0.0000.5207.

Paciente E.R.S, gênero masculino, 55 anos, apresentou-se no ano de 2014 ao consultório odontológico tendo sido encaminhado pelo médico otorrinolaringologista para iniciar o tratamento com o AIO. O paciente informou que não estava conseguindo utilizar o CPAP regularmente, pois o achava incômodo de usar, por isso procurou no AIO uma alternativa para o tratamento da AOS. Na anamnese informou ter a apneia obstrutiva do sono, ronco alto, fibromialgia, relatou ser muito estressado, sente sono e cansaço durante o dia, tem vontade de continuar dormindo (sono não restaurador), sudorese excessiva durante o sono, lapsos de memória, salivação excessiva, cefaleia quando acorda e hipertensão arterial (170/110 mmhg).

Todo o tratamento assim como sua divulgação foi devidamente autorizado e registrado pelo paciente no termo de consentimento livre esclarecido. Não foi possível realizar a PSG basal do paciente, pois o mesmo ainda estava utilizando o CPAP mesmo não aprovando a terapia, logo havia a possibilidade de os resultados estarem alterados. Na consulta inicial foi observada boa condição oral, sendo escolhido o AIO de avanço mandibular para iniciar o tratamento.

As polissonografias noturnas completas (PSG) foram realizadas em dois diferentes laboratórios do sono e com o AIO em boca. No primeiro laboratório, a PSG foi realizada em 04/08/2014, com o equipamento Poliwin $\AA$, tendo como parâmetros eletrofisiológicos: EEG, EMG submentoniano; EMG tibial anterior (E e D); sensor de ronco; fluxo aéreo nasal; cintas torácicas e abdominal; oxímetro de pulso e ECG; e filmagem com vídeo digital. No outro laboratório o segundo exame foi realizado em 10/08/2015 e o terceiro em 27/01/2016, ambos em aparelho digital (Alice 5 - Respironics), tendo como parâmetros: EEG, EOG, ECG, EMG (de mento e tibiais), fluxo aéreo nasal, fluxo aéreo naso-bucal, cintas torácicas e abdominal, sensor para ronco, monitor de posição e oxímetro de pulso.

O aparelho foi confeccionado em resina acrílica termo polimerizável e com um disjuntor palatino adaptado entre as duas bases de acrílico, de forma que quando é acionado, avança a placa mandibular. Foi construído com aproximadamente $50 \%$ da protrusão mandibular máxima do paciente. Os acompanhamentos das sessões foram semanais no primeiro mês, quinzenalmente no segundo mês e uma vez por mês nos meses seguintes.O AIO foi colocado inicialmente em posição de topo, após uma semana o mesmo retornou relatando não ter notado uma grande melhora dos sintomas, foi então realizado o avanço de $1 \mathrm{~mm}$ da placa mandibular (Figuras 1, 2 e 3).

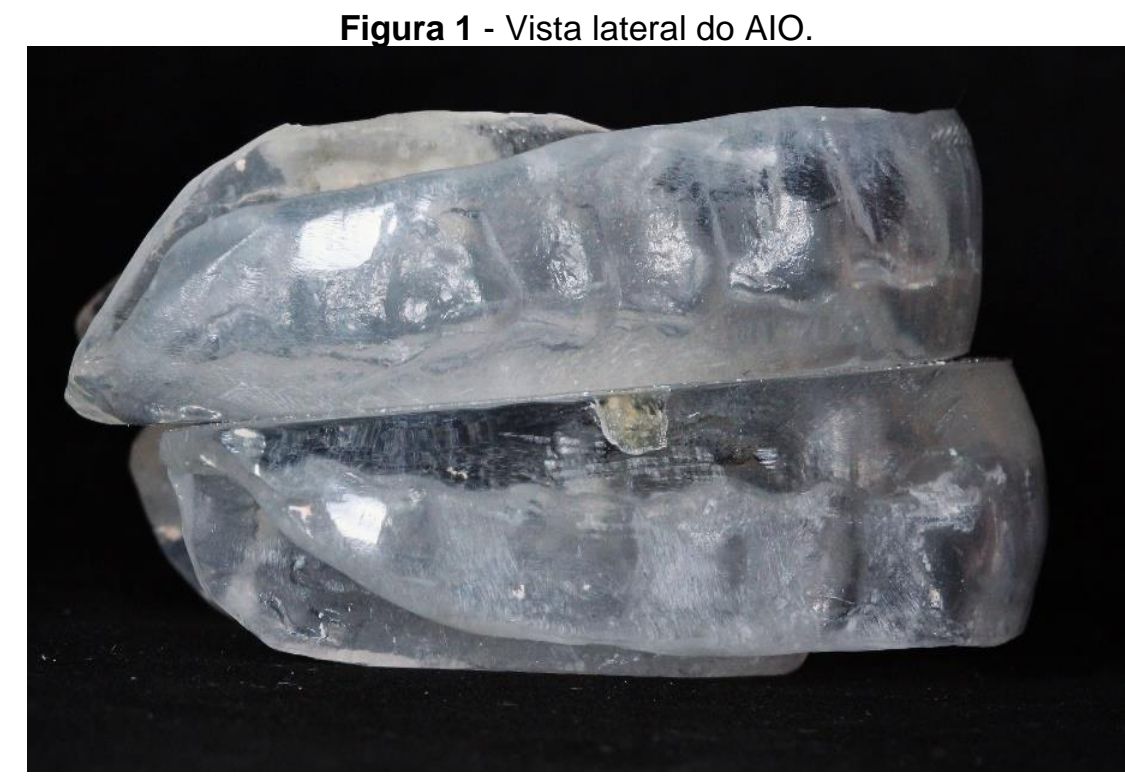

Fonte: Santiago JB, et al., 2020. 
Figura 2 - Vista axial do AIO.

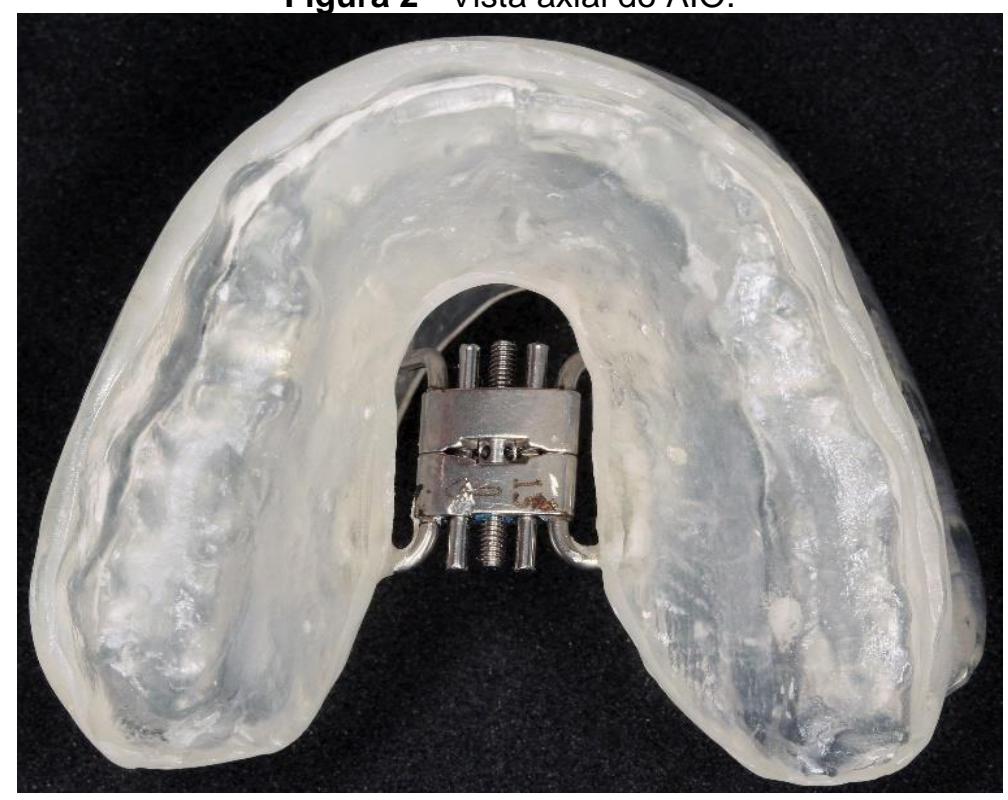

Fonte: Santiago JB, et al., 2020.

Figura 3 - Miniatura do AIO instalado em modelos de resina acrílica.

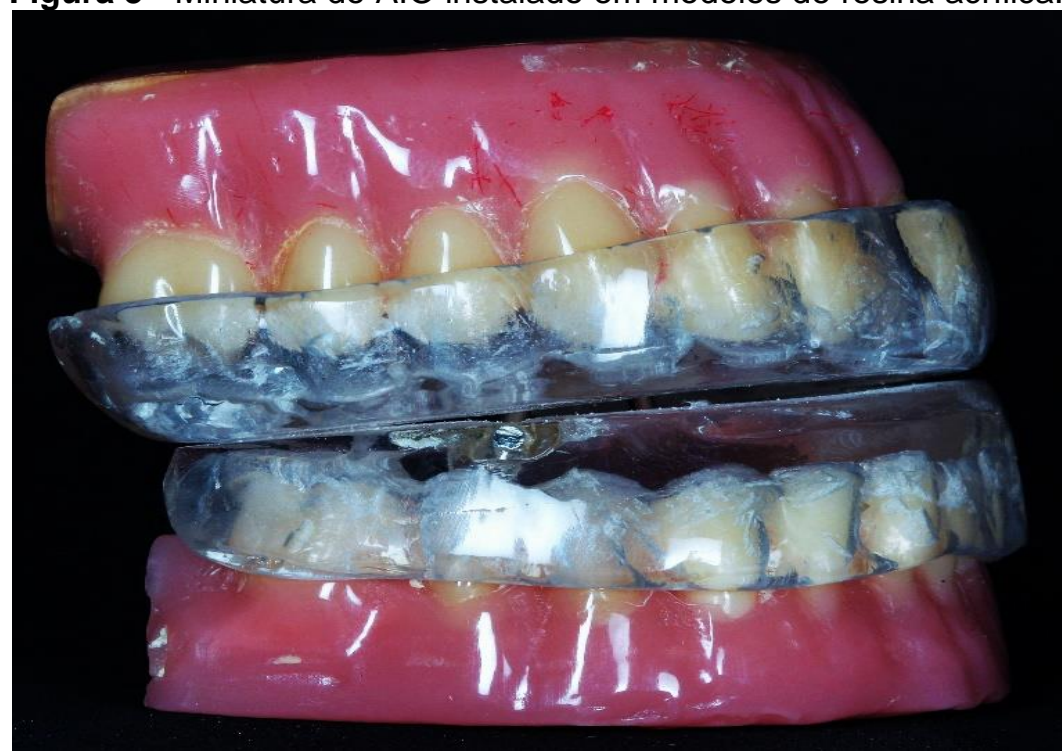

Fonte: Santiago JB, et al., 2020.

O AIO foi adequadamente ajustado acarretando na resolução dos sintomas. O paciente se manteve assíduo as consultas de controle e não houveram queixas com relação a musculatura mastigatória ou articulação temporomandibular. Cerca de um ano e seis meses após o início do tratamento houve melhora dos parâmetros analisados, e principalmente a redução do IAH para uma condição de normalidade (Tabela 1).

Tabela 1 - Resultados das polissonografias.

\begin{tabular}{cccc}
\hline Parâmetros Avaliados & PSG & PSG & PSG \\
ago/14 & ago/15 & jan/16 \\
\hline IAH & $11,26 / \mathrm{h}$ & $7,4 / \mathrm{h}$ & $3,9 / \mathrm{h}$ \\
\hline Saturação média/ mínima de oxihemoglobina $(\%)$ & $91 / 70$ & $96 / 91$ & $96 / 87$ \\
\hline IMC $\left(\mathrm{Kg} / \mathrm{m}^{2}\right)$ & 24.96 & 23,97 & 24,65 \\
\hline Índice de microdespertares & $7,87 / \mathrm{h}$ & $13,4 / \mathrm{h}$ & $7,6 / \mathrm{h}$ \\
\hline Eficiência do sono & $89 \%$ & $92,8 \%$ & $92,1 \%$ \\
\hline
\end{tabular}

Fonte: Santiago JB, et al., 2020. 
Passado esse período foi aplicado um questionário de opinião (sem validação), criado especificamente para esse estudo, onde o paciente pôde responder algumas perguntas acerca das terapias. $O$ mesmo informou que passou a ter sono reparador, aumento na qualidade de vida, mais disposição para realizar suas atividades laborais, ausência de ronco e uma característica marcante foi a diminuição das dores musculares consequentes da fibromialgia (Quadro 1).

Quadro 1 - Questionário comparativo sobre o uso do AIO e CPAP.
1. Como era a sua qualidade de vida antes do uso do aparelho intra oral e o CPAP?
(X) Péssima
( ) Ruim
( ) Normal
( ) Boa
( ) Excelente
2. Durante o uso do CPAP você notou alguma melhora do seu quadro clínico?
(X) Sim
3. Caso tenha respondido "Sim", como seria essa melhora em uma escala de 0 a 10 ? (Sendo "0" igual a nenhuma melhoria, e "10" igual a uma melhora completa). Assinale com um " $X$ ".
$\begin{array}{lllllllllll}\text { ( ) } 0 & \text { () } 1 & \text { (X) } 2 & \text { ( ) } 3 & \text { ( ) } 4 & \text { ( ) } 5 & \text { ( ) } 6 & \text { ( ) } 7 & \text { ( ) } 8 & \text { ( ) } 9 & \text { ( ) } 10\end{array}$
4. Durante o uso do aparelho intra oral você notou alguma melhora do seu quadro clínico?
(X) Sim
5. Caso tenha respondido "Sim", como seria essa melhora em uma escala de 0 a 10 ? (Sendo "0" igual a nenhuma melhoria, $e$ "10" igual a uma melhora completa). Assinale com um " $X$ ".
$\begin{array}{lllllllllll}\text { () } 0 & \text { () } 1 & \text { () } 2 & \text { () } 3 & \text { () } 4 & \text { ( ) } 5 & \text { () } 6 & \text { (X) } 7 & \text { () } 8 & \text { () } 9 & \text { () } 10\end{array}$
6. Durante o uso simultâneo do aparelho intra oral e do CPAP, você notou alguma melhora do seu quadro clínico?
( ) $\operatorname{Sim}$
(X) Não

7. Caso tenha respondido "Sim", como seria essa melhora em uma escala de 0 a 10? (Sendo "0" igual a nenhuma melhoria, e "10" igual a uma melhora completa). Assinale com um " $X$ ".
( ) 0
( ) 1
( ) 2
( ) 3
( ) 4
( ) 5 ( ) 6
( ) 7 ( ) 8
() 9 ( ) 10

8. Em uma escala de 0 a 10, quão incômodo foi utilizar o CPAP? (Sendo "0" igual a nenhum incômodo, e "10" igual ao maior incômodo possível). Assinale com um " $X$ ".

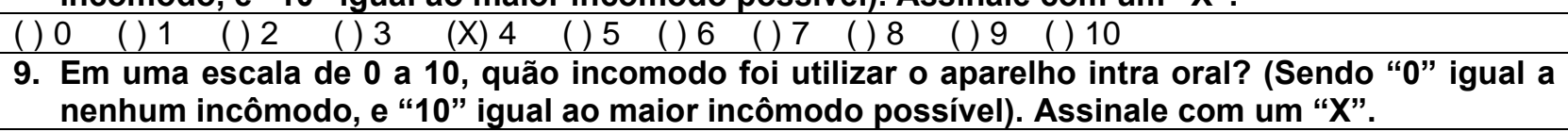
$\begin{array}{lllllllllll}(\text { () } 0 & \text { (X) } 1 & \text { () } 2 & \text { () } 3 & \text { () } 4 & \text { () } 5 & \text { () } 6 & \text { () } 7 & \text { () } 8 & \text { () } 9 & \text { () } 10\end{array}$

10. Em qual etapa do tratamento você sentiu que teve uma melhora mais significativa do seu quadro clínico?

( ) Quando utilizei apenas o CPAP

(X) Quando utilizei apenas o aparelho intra oral

( ) Quando utilizei concomitantemente o aparelho intra oral e o CPAP

11. Após o tratamento com os aparelhos, como você classifica a sua qualidade de vida agora?

( ) Péssima

( ) Ruim

( ) Normal

(X) Boa

( ) Excelente

Fonte: Santiago JB, et al., 2020.

O paciente teve câncer de próstata e após o uso do aparelho houve uma redução da noctúria, com isto melhorando também a qualidade do sono. As vezes em que o paciente precisou realizar reparos no aparelho, ficando algumas noites sem uso do mesmo, relatou que após os primeiros dias os sintomas retornaram. 


\section{DISCUSSÃO}

Pelo fato do tratamento da AOS com AIO ser de longa duração, alguns efeitos colaterais, como desconfortos ou dores na região orofacial, podem surgir interferindo diretamente na continuidade do tratamento pelos pacientes e impossibilitando a manutenção dos resultados alcançados pelo aparelho.

Algumas das principais causas da não adesão ao tratamento com o AIO, em ordem decrescente, são: o fato de alguns pacientes o acharem "incômodo de usar", por surtir "pouco ou nenhum efeito", "desconforto ou dor dentária", "dificuldade para dormir", "má adaptação após tratamento odontológico" e "desconforto ou dor gengival" (NISHIGAWA K, et al., 2017).

No presente estudo, o paciente sempre compareceu às consultas de controle e nunca referiu nenhum desconforto oriundo do tratamento, nem mesmo no período de adaptação, afirmando que só não utilizou o AIO nos dias em que o mesmo estava sendo ajustado em laboratório, e nesses períodos os sintomas da AOS retornaram.

O exame eleito para o diagnóstico da AOS é a PSG de noite inteira, a qual avalia uma série de parâmetros fisiológicos que determinam o grau de severidade da apneia, auxiliando na escolha da terapia mais adequada (CAMPOSTRINI DDA, et al., 2014). O IAH foi escolhido como referência para acompanhar o tratamento do paciente por ser o índice mais utilizado na literatura, atuando no registro dos períodos de apneia e hipopneia por hora de sono.

As visitas de acompanhamento e as polissonografias ou exames de monitoramento domiciliar para detecção de AOS, devem receber a devida atenção (GUIMARÃES MLR, et al., 2018; RAMAR K, et al., 2015). A partir dos resultados destes exames é possível avaliar a eficácia a longo prazo da terapia. Neste relato, 0 paciente apresentou uma ótima adesão (8h por dia, durante 7 dias por semana) ao tratamento com AIO, realizou todas as visitas de acompanhamento e realizou todas as polissonografias de controle, o que possibilitou a análise constante dos exames e consequentemente um melhor controle da AOS.

Consecutivas dessaturações da oxihemoglobina consequentes da AOS ocasionam o agravo da insuficiência do miocárdio por conta do aumento na pós-carga do ventrículo esquerdo; e elevação da frequência cardíaca e pressão sanguínea ocasionadas pelos despertares e aumento da atividade do sistema nervoso simpático (BRADLEY TD e FLORAS JS, 2000).

Os níveis de saturação média de oxihemoglobina do paciente, obtidos nas polissonografias com o AIO em boca, apresentaram-se normais e com aumento de $5 \%$ nos dois últimos exames, mostrando que as vias aéreas superiores tiveram sua permeabilidade aumentada por conta do uso do $\mathrm{AIO}$, dessa forma reduzindo os riscos sistêmicos e cardiovasculares (ALENCAR HBB, et al., 2016; GODOLFIM LR, 2010).

A obesidade é um fator de risco reversível para a AOS e está presente em cerca de $70 \%$ dos pacientes apneicos, por tanto, deve ser levada em consideração para o tratamento da AOS (VIEIRA CIV, et al., 2011). A predominância da AOS em indivíduos com obesidade mórbida pode chegar a $80 \%$ e $50 \%$ nos gêneros masculino e feminino, respectivamente (NABARRO PAD e HÖFLING RTB, 2008).

Segundo a Associação Brasileira para o Estudo da Obesidade e da Síndrome Metabólica (ABESO), um indivíduo com o IMC entre 18,5 e 24,9 é considerado normal ou eutrófico com grau zero de obesidade. O IMC do paciente, em toda a etapa da pesquisa, se manteve em níveis normais, favorecendo dessa forma um melhor resultado final da terapia (ABESO, 2016)

Alguns indicadores auxiliam na identificação de quais pacientes possuem um melhor prognóstico para o tratamento, porém eles ainda não são totalmente confiáveis na prática clínica. Neste trabalho o paciente tinha 55 anos, possuía o IMC normal e IAH baixo. Com exceção da idade, o IMC e o IAH vão de encontro ao que é reportado na literatura, ou seja, pacientes com menor IAH e IMC possuem um melhor prognóstico, assim como pacientes com menor idade (DIELTJENS M, et al., 2013; SUTHERLAND K, et al., 2015).

O fato do paciente não ser jovem e mesmo assim ter tido sucesso na terapia com o AlO, reduzindo de uma apneia leve a ponto de chegar a um quadro de normalidade sem nenhum tipo de intercorrência, faz surgir um questionamento acerca da confiabilidade dos fatores que predizem os indivíduos que possuem um melhor 
prognóstico para a terapia com o aparelho intra oral, e ao mesmo tempo evidencia a importância da condução de pacientes ao tratamento por profissionais do sono, mesmo que esses apresentem indicadores negativos.

Quando se faz uma comparação entre o AIO e o CPAP a respeito da qualidade de vida proporcionada por esses aparelhos, é observada a superioridade do AIO em inúmeros pontos, como no isolamento social, na mobilidade física, na dor, na função emocional e no sono. De fato, o CPAP tem a capacidade de redução do $\mathrm{IAH}$ superior ao $\mathrm{AIO}$, porém, de acordo com vários estudos, há uma melhora significativa da qualidade de vida dos pacientes com AOS que utilizam o aparelho intra oral (GAGNADOUX F, et al., 2009).

A partir das polissonografias foi possível adquirir os registros positivos durante o tratamento, assim como a opinião do paciente que foi registrada através do questionário, no qual mostrou que houve uma preferência do mesmo pelo AIO ao invés do CPAP. Através da PSG e do relato do paciente, pôde-se analisar uma melhora na qualidade de vida de forma gradual, o que permitiu, com normalidade, o retorno do mesmo as suas atividades diárias.

A eficiência do sono do paciente se manteve sempre acima do valor normal de referência (acima de $85 \%$ ), caracterizando um aumento do tempo de sono do paciente, uma vez que é calculada a partir da divisão do tempo total de sono pelo tempo total de registro (TTS / TTR) (TOGEIRO SMGP e SMITH AK, 2005).

O tratamento para controle da AOS tem como objetivo a eliminação ou redução dos eventos respiratórios obstrutivos, desta forma restaurando o sono e a oxigenação arterial até um padrão de normalidade, corrigindo alterações neuropsíquicas e cardiovasculares (DAL-FABBRO C, et al., 2010). O tratamento é considerado um sucesso quando se reduz, pelo menos, $50 \%$ do IAH (GODOLFIM LR, 2010; BITTENCOURT LRA e PALOMBINI LO, 2008).

Neste caso, segundo os registros polissonográficos, no início do tratamento o paciente apresentava uma AOS leve $(\mathrm{IAH}=11,26 / \mathrm{h}$ ) e passou para um quadro de normalidade (IAH = 3,9/h), consequentemente melhorando os sintomas, dessa forma o tratamento escolhido foi considerado satisfatório. É válido ressaltar que tanto o CPAP quanto o AIO possuem indicações e contraindicações precisas, não sendo terapias excludentes e nem complementares, o que de fato determina o melhor método é o grau da AOS e a tolerância do paciente a terapia escolhida.

Após a avaliação dos exames complementares realizados e respostas do questionário (comparativo do $C P A P$ versus $\mathrm{AIO}$ ) preenchido pelo paciente, pode-se confirmar que o AIO foi eficaz e promoveu: redução do IAH; Ausência da AOS (durante o uso do AIO); Níveis de saturação média de oxihemoglobina acima do mínimo esperado; Índice de microdespertares estável contribuindo para uma boa eficiência do sono.

Com todas essas mudanças nos índices polissonográficos, pode-se dizer que o paciente está com a AOS controlada, ocasionando melhoras significativas na qualidade de vida do indivíduo, na sua condição sistêmica, na qualidade do sono, na respiração noturna e sonolência diurna. Dessa forma, o paciente voltou a desempenhar suas atividades cotidianas normalmente, devolvendo a este não apenas uma melhor condição de saúde física e mental, mas uma melhor reintegração social.

\section{REFERÊNCIAS}

1. ALENCAR HBB, et al. Tratamento da Síndrome da Apneia Obstrutiva do Sono com o Uso Supervisionado de Aparelhos Intraorais: Relato de Caso. Ensaios e Ciênc: C. Biológicas, Agrárias e da Saúde, 2016; 20(2):59-64.

2. Associação Brasileira para o Estudo da Obesidade e da Síndrome Metabólica. Diretrizes Brasileiras de Obesidade; 2016.

3. BITTENCOURT LRA. Abordagem geral do paciente com síndrome da apneia obstrutiva do sono. Rev Bras Hipertens 2009; 16(3):158-63.

4. BITTENCOURT LRA, PALOMBINI LO. Síndrome da apneia e hipopneia obstrutiva do sono: fisiopatologia. In: Tufik S. (Org.). Medicina e biologia do sono. São Paulo: Manole, 2008; p.240-7.

5. BRADLEY TD, FLORAS JS. Pathophysiological interactions between sleep apnea and congestive heart failure. In: Bradley TD, Floras JS. Sleep apnea implications in cardiovascular and cerebrovascular disease. Lung Biology in health and Disease, New York: Marcel Dekker, 2000;146: 385-414.

6. CAMPOSTRINI DDA, et al. Síndrome da apneia obstrutiva do sono e doenças cardiovasculares. Rev Neurociências, $2014 ; 22(1): 102-12$. 
7. DAL-FABBRO C, et al. Classificação dos distúrbios do sono. In: Dal-Fabbro C, Chaves Junior CM, Tufik S. Aodontologia na medicina do sono. Maringá: Dental Press,2010; 1: 65-98.

8. DEY D, et al. Obstructive sleep apnoea detection using convolutional neural network based deep learning framework. Biomedical engineering letters, 2018; 8(1):95-100.

9. DIELTJENSM, et al. Objectively measured vs self-reported compliance during oral appliance therapy for sleepdisordered breathing. Chest, 2013; 144(5):1495-502.

10. GAGNADOUX F, et al. Titrated mandibular advancement versus positive airway pressure for sleep apnoea. Eur Respiratory 2009; 34(4):914-20.

11. GUIMARÃES MLR, et al. Severe obstructive sleep apnea treatment with mandibular advancement device: A case report. Sleep Science, 2018; 11(2):118.

12. GODOLFIM LR. Distúrbios do sono e a odontologia: tratamento do ronco e a apneia do sono. 1. ed. São Paulo: Santos, 2010; p236.

13. ITO FA, et al. Condutas terapêuticas para tratamento da síndrome da apnéia e hipopnéia obstrutiva do sono (SAHOS) e da síndrome da resistência das vias aéreas superiores (SRVAS) com enfoque no aparelho anti-ronco (AAR-ITO). Revista Dental Press de Ortodontia e Ortopedia Facial, 2005; 10(4):143-56.

14. NABARRO PAD, HÖFLING RTB. Efetividade do aparelho ortopédico Bionator de Balters no tratamento do ronco e apnéia do sono. Revista Dental Press de Ortodontia e Ortopedia Facial, 2008; 13(4):36-44.

15. NEVES GSML, et al. Transtornos do sono: visão geral. Rev Bras Neurol,2013; 49(2):57-71.

16. NISHIGAWA K, et al. Complications causing patients to discontinue using oral appliances for treatment of obstructive sleep apnea. Prosthodontic Research, 2017; 61(2):133-8.

17. PACHECO FYR, et al. Síndrome da apnéia/hipopnéia obstrutiva do sono: artigo de revisão bibliográfica. UNILUS Ensino e Pesquisa, 2016; 12(29):45-52.

18. RAMAR K, et al. Clinical practice guideline for the treatment of obstructive sleep apnea and snoring with oral appliance therapy: an update for 2015: an American Academy of Sleep Medicine and American Academy of Dental Sleep Medicine clinical practice guideline. Journal of clinical sleep medicine, 2015; 11(7):773-827.

19. SAGLAM-AYDINATAY B, TANER T. Oral appliance therapy in obstructive sleep apnea: Long-term adherence and patients' experiences. Medicina oral, patologia oral y cirugia buccal, 2018; 23(1): 72.

20. SILVA GA, PACHITO DV. Abordagem terapêutica dos distúrbios respiratórios do sono tratamento com ventilação nãoinvasiva (CPAP, BiPAP E AUTO-CPAP). Medicina (Ribeirao Preto. Online),2006;39(2):212-7.

21. SUTHERLAND K, et al. Oral appliance treatment for obstructive sleep apnea: an update. Journal of Clinical Sleep Medicine, 2014; 10(2):215-27.

22. VIEIRA CIV, et al. Tratamento da sindrome da apnéia e hipopnéia obstrutiva do sono (sahos) com aparelhos intrabucais. Ortodontia, 2011; 44(2):157-64.

23. SUTHERLAND K, et al. Oral appliance treatment response and polysomnographic phenotypes of obstructive sleep apnea. Clin Sleep Med, 2015; 11(8):861-8.

24. TOGEIRO SMGP, SMITH AK. Métodos diagnósticos nos distúrbios do sono Diagnostics methods for sleep disorders. Rev Bras Psiquiatr, 2005; 27(Supl I):8-15. 